\title{
Personas in Cross-Cultural Projects
}

\author{
Lene Nielsen \\ Snitker \& Co., Bredgade 23A, 2 \\ 1260 Copenhagen, Denmark \\ lnasnitker.com
}

\begin{abstract}
Personas are a method to communicate data about users and to aid in the perception of users. The method is supposed to create a shared perception of the users that is not built on preconceived ideas, but on field data. The paper presents an experiment where the same persona description was sent to 16 participants in 9 countries. The participants were asked to return a photo that resembled the persona and explain their choice. Analysis of the photos and the explanations show that there is a difference between the participants with professional experiences and those without. The experienced tend to interpret the text and use people in their own immediate surroundings in the explanation for choosing the photo. The second group tends to find exact words and use these as explanation. The photos they choose are of stereotypical business-persons. The different strategies might hinder engagement in the persona.
\end{abstract}

Keywords: Personas, Scenarios, Culture.

\section{Introduction}

Personas are considered a method to communicate data about users and to aid in the perception of users. Instead of project participants having individual understandings, the method is supposed to create a shared perception of the users that is built on field data and not on preconceived ideas. There is consensus that a persona is presented as a text with an accompanying illustration of the persona either as a photo or a drawing [1]. This paper investigates how different users from different cultures perceive the same textual description of a persona. The paper presents a follow-up to an earlier study presented at a workshop at NordiChi 2008 [2].

As all communication products personas presentations have two parts involved - a sender who writes the persona presentation and a receiver who reads and perceives the persona. The personas presentations have commonalities with stories, and as applies to stories, personas presentations can never be told in their entirety - some information the reader will have to infer. When trying to make meaning of a story, the reader will fill in the narrative gaps in the text [3], with information from the reader's own personal experiences. This goes for personas descriptions as well, where the description cannot present all information about the persona and the reader infers the missing information in the meaning making process. The information that is not received as direct story elements, the reader infers from expectations, knowledge of the depicted domain, and own cultural background [4]. Storytelling is a common experience, but the reception of a story is individual and each reader creates an individual story. 
In connection to personas descriptions the question becomes: do culturally different readers fill the narrative gaps differently? And if so, does this impact the application of the personas method in practice?

\section{The Experiment}

In the following I will report the findings from two studies of how people from different countries perceive a persona description. The first study [2] involved six participants

\section{$\mathbf{X}$, manager of online marketing.}

$\mathrm{X}$ is 42 and married, with a son and a daughter. $\mathrm{X}$ lives in a house a bit outside the capital and commutes to and from work.

At work, $\mathrm{X}$ likes to wear formal clothes, but not too formal. $\mathrm{X}$ tries to do a bit of fitness, but finds it hard to get it into the busy schedule with work and family.

$\mathrm{X}$ is content with the place in career and has not, as in the youth, the possibilities to work long hours as X wants to be with the family "I want to play with my children every day". Sometimes $\mathrm{X}$ works in the evening, when the kids are in bed.

\section{Education}

$\mathrm{X}$ has a degree in sales- and marketing from a business school. $\mathrm{X}$ studied before there was anything called online marketing, therefore $\mathrm{X}$ has the knowledge on online marketing from books and seminars.

\section{The company}

$\mathrm{X}$ is employed in a large company with a long tradition of offline marketing. $\mathrm{X}$ is the manager of the online department in the company and is responsible for the development of the company's online initiatives.

$\mathrm{X}$ is responsible for the company's online ads. Here $\mathrm{X}$ has to make sure that the campaigns support the company's offline activities. $\mathrm{X}$ is also responsible for getting clients for the department's new activities. $\mathrm{X}$ sees a huge benefit in online marketing, as it is easy to access what you get you for your money.

$\mathrm{X}$ makes sure that the company's website is constantly updated and that new initiatives make it interesting for the customers to visit the website regularly.

$\mathrm{X}$ experiences from time to time that the organization is sluggish and that the road from idea to action can be long.

\section{The online department}

The online department employs four people. It is primarily $\mathrm{X}$ who is responsible for the company's online marketing. The other employers are mainly engaged in the website and how recently added elements perform. It is important for the department to show a high level of activity on their new initiatives.

\section{Communication}

$\mathrm{X}$ is in daily contact with the company's many media- and advertising agencies about campaign activities for the next quarter. X lets the media agencies deliver media plans. "I do not have the time to keep an eye on what sites to post on. They are much better at that." X has the final say on the media plans.

$\mathrm{X}$ reports directly to the board and presents last quarter's result as well as new initiatives. This is done by collecting numbers from the agency. They are copied into Excel and later into a PPT.

\section{Technology}

Apart from X, everybody in the online department is interested in technology. X finds new technologies expensive and difficult to handle, but has a notion that there might be some benefits.

Fig. 1. Persona description 
from India, China and Denmark. All participants except one were young and either MA or PhD students. This study was followed by a second study with nine usability professionals from Japan, Brazil, France, Holland, Russia, New Zealand, Germany and USA. All were familiar with the persona method.

\subsection{Method}

As can be seen in Fig. 1, the persona description portrayed a person working with marketing. The description was written with an attempt to remove cues regarding age, gender, or culture. In both studies the participants were asked to: 1) read a persona description 2) find a photo on the internet that resembled the persona; 3 ) write a short explanation why they had chosen this particular photo; 4) mail both the photo and explanation to me.

When the photos and the written explanations arrived, the texts were analyzed in order to investigate the connection between the photos and the explanations the participants each gave for choosing them. The analysis focused on strategies for finding photos, explanations, and visual appearances.

In the first study the age of the persona's children were mentioned (age 3 and 7), but it turned out that their age skewed the perception towards a westerner, e.g. in India the average age of people becoming parents is lower than it is in Denmark. In the second study the age of the children were removed from the description.
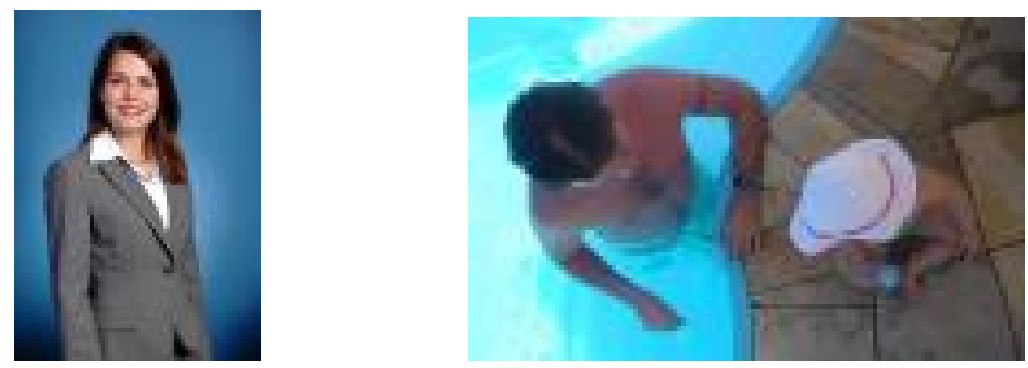

Fig. 2. The two photos below illustrate suggestions for the persona from the first and second study, respectively

\section{Strategies for Finding Photos}

\subsection{Cues or Interpretations}

In both studies there seemed to be two strategies at play when the participants chose a photo; either looking for specific words that could function as cues for the choice of photo or reading the text and providing an argument that had an interpretation of the text as point of departure. As can be seen in Table 1 most participants chose a strategy based on interpretation and, with one exception, the participants who chose the strategy of finding exact wordings in the description were students. 
Table 1. The informants' strategies

\begin{tabular}{|c|l|}
\hline Strategy based on description & \multicolumn{1}{|c|}{ Strategy based on interpretation } \\
\hline $\mathrm{CH} 2, \mathrm{DK} 1, \mathrm{DK} 2, \mathrm{DK} 3, \mathrm{IN} 2, \mathrm{IN} 3, \mathrm{RU}$ & $\begin{array}{l}\text { CH1, CH3, IN3, NL, US1, US2, FR, } \\
\text { BRA, JP }\end{array}$ \\
\hline
\end{tabular}

In the strategy based on description the participants found a specific description in the text that served as explanation for the choice of photo. 'She has young children and therefore she could not be old' (Danish informant 2). 'He has no time to do much exercise, so he may be a little fat' (Chinese informant 2). "The concern about fitness and family made me think your persona looks more like a woman than a man. So I was looking for a grown-up business woman." (Russian Informant).

In the interpretation strategy the participant interpreted the text and used this as an explanation for the choice of photo 'I don't know why but I tend to associate obesity with reluctance to new technology mindset. Don't ask me why!'(Indian informant 3). 'Information Technology means he would earn more money than average' (Chinese informant 1).

It applies to both strategies that when the readers have previous knowledge of the depicted domain, they use their own background in trying to understand the persona description. They do so by reaching out for persons in the intimate surroundings and relates these to the persona description. 'I assume I know the person, because of my previous career and involvement in advertising. I, from the get go, had an idea of how I presumed this person to look, since to me we all looked alike at the agency after a while. I took inspiration from my former superiors and my general ideas of how people in his position dress and tend to appear physically.'(Danish Informant 1). 'He has a French look (he looks like my brother in law who has similar position in a company).' (French Informant).

In the description below, the argument for a photo can be followed.

"When reading the persona description I concluded this could both be a male or female persona. Based on some elements in the text I might be more inclined to make it a male, but then again (being the son of a working mother), there is nothing that will not make it possible in the Netherlands to let this persona be a woman. I sort-of randomly browsed Flickr.com in search for my Karin and Simon. I did not want to use stock images, I wanted to show them in a more natural surroundings. The images of Karin commuting (and working) made that image suitable. Simon doing something with his kids made that one suitable. Again, I didn't look for people in the working environment, wearing suit and tie. There needed to be some additional element to it that gave it a bit more richness, something to make them more real. Again, I felt Simon to be boring and the man in the image doesn't look all to excited. Karin feels more adventurous and driving and being on the phone is as exciting as it gets..." (Dutch Informant).

The participant starts off by picking a name, then adds information from the text, and ends by an elaborate interpretation that has to do with gender. Here the participant relates the discussion of gender to his own upbringing, and argues that he is unable to make a decision whether the description was of a male or a female, but adds values to 
the gender - if the description is of a man, he is a bore. If it is of a woman, she is adventurous. The description reflects both on the process of reasoning and the workflow that can be described as: decide name - go to Flickr - search photos with a set of criteria.

\subsection{Stereotypes}

The Dutch informant draws on his previous knowledge of people in a position similar to the persona description he forms categories of men who want to spend time with children and women at work, and adds the values of being boring and adventurous to the categories. These can be seen as individual categories. He also supplements with an awareness of wanting to present something other than the social stereotypical image of a business person in suit and tie. This is in line with the construction of stereotype as both individual and social constructs. The individual construction occurs in meetings with new persons, where we do not see them as unique persons, but add them to previous formed categories, built on knowledge of previous meetings with others - and often expressed as stereotypes [5]. Schutz distinguishes between the experiences of earlier meetings and the stereotype as a generalizations of social reality, the latter being general and empty of content [6].

In the first study the stereotypes were mainly socially constructed, it was observed that all, except one, of the participants picked a photo of a person in a business suit, even the only photo of a female, and that most participants chose a photo of a person with a non local appearance. Five participants from Denmark, India and China chose photos of western looking business persons, the rest of the participants chose photos of locals (the female Danish participant chose a person with a non-Danish appearance). In this choice there seems to be a socially constructed and stereotypical comprehension of how a business person looks - despite culture.

The individually constructed stereotypes could be seen in comments such as "I tend to associate obesity with reluctance to new technology".

In the second study only one chose a photo of a business stereotype and all participants chose photos of people with a local appearance.

The rationale behind this difference can be explained with a difference in familiarity with the area described, "The anonymity of typification is inversely proportional to fullness of content" [6] p 80. As the students do not have many and varied experiences with people working in marketing, they tend to fall back on an empty and socially formed category of how business people look. The usability consultants have had encounters with people from marketing this dissolves the stereotype and initiates that the consultant expresses the persona as an individual.

\subsection{Age}

The analysis showed that when the participants had an individually formed category based on experiences they chose the age of the persona based on their own age. These photos were mixed in appearance and in general they portrayed persons older than in the first study. In this study the photos depicted younger persons. The Danish participants chose photos of people even younger than those chosen by the other student participants. 


\subsection{Gender}

Analysis of both studies shows that the participants' gender influences the choice of photo. Of the 16 responses only four had chose a depiction of a woman and, as mentioned, of these one was undecided whether it was a woman or a man. The three participants, who had a clear vision of the gender as being a woman, were all female.

There also seems to be gender stereotypes at play. When men are depicted they are more often shown in surroundings with their children as the persona is described as one who cares for the family. When women are depicted they are always shown in business surroundings.

\section{Conclusion}

In the earlier study [2] there seemed to be a difference between easterners and westerners as all of the participants with a western background chose a strategy based on description. In this study that added experienced participants from all over the world the conclusion moved into a new direction and the study have findings that might influence the personas method. The study found differences in the constructions that seem to be related to the two different perception strategies; interpreting or looking for clues in the description. Interpreting seems to be linked to individual constructions, and looking for clues seems to be connected to the socially constructed stereotypes.

Social constructions, stereotypes, are characterized by being empty of content, are seen when the participants have less professional experience and are unable to add content to their understanding of the person. Individual constructions are observed when participants have more professional experience. The professional participants generate constructions based on individual predefined attitudes. These participants use their own background to fill in the details such as age and knowledge of the domain, and of how a person in marketing looks. Individual constructions are also formed of arbitrary reasons that have to do with the participants' own internal logics such as obesity links to technology resistance, professional women has to be shown in professional settings, etc.

A difference in the respondent group that might explain the variation in strategies and constructions, can be a diversity in experiences - the younger group without professional experiences tend to reach for a strategy that involves looking for exact words in the text and the participants with professional experience tend to interpret the text and use previous knowledge of the depicted area. The reader tries to understand the persona description by reaching out for persons in the intimate surroundings thereby relating to the persona description.

The initial research question was: do culturally different readers fill the narrative gaps differently? The answer to this question is that this cannot be observed from this study. It rather seems to be differences in experience that create the variation in strategies and explanations.

The second part of the question was: And if so, does it impact the application of the personas method in practice? The obvious answer to this question would be "no", but both the strategies and the construction can be obstacles to one of the goals of 
using personas: to create an empathic understanding of the user that can serve as a catalyst for design ideas [7] as most participants seem to create either individual or social stereotypes.

In this experiment there were no apparent variations when looking at the respondents culture as the focus area is well-known; a description of a business persona is quite familiar to most, but more unfamiliar personas in more unfamiliar surroundings might create comprehension strategies that might create even greater obstacles for a necessary persona engagement.

To determine the impact on cross-cultural personas projects, further and more detailed studies need to be conducted where the persona description is moved away from the participants' immediate area of experience.

Acknowledgements. I want to thank the CultUsab project, UX Alliance, and all the participants.

\section{References}

1. Pruitt, J., Adlin, T.: The Persona Lifecycle: Keeping people in mind through-out product design. Morgan Kaufmann, San Francisco (2006)

2. Nielsen, L.: Different Cultures' Perception of Personas Descriptions. Cultural Usability and Human Work Interaction Design - techniques that connects. In: Proceedings from NordiCHI 2008 Workshop Sunday, October 19, pp. 43-46 (2008)

3. Iser, W.: The reading process: a phenomenological approach. In: Wood, N. (ed.) Modern Criticism and Theory: a reader, pp. 188-205. Pearson Education, Harlow (2000)

4. Bordwell, D.: Narration in the Fiction Film. Routledge, London (1997)

5. Macrae, N.C., Bodehausen, G.V.: Social Cognition: Categorical person perception. British Journal of Psychology 92, 239-255 (2001)

6. Schutz, A., Luckmann, T.: The Structures of the Life-World. Northern University, Illinois (1973)

7. Nielsen, L.: Engaging Personas and Narrative Scenarios. Samfundslitteratur, Copenhagen (2004) 